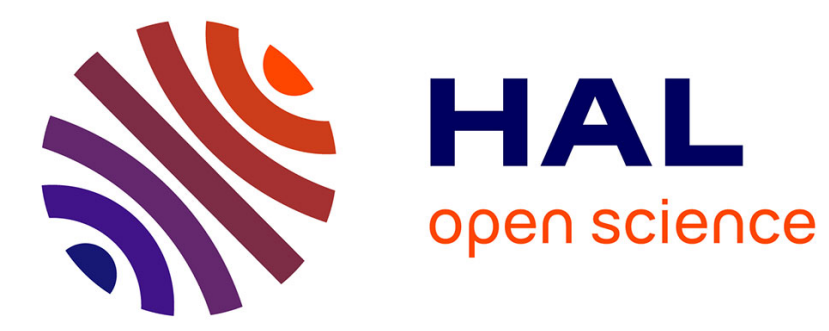

\title{
Radiative heat transfer between metallic nanoparticle clusters in both near field and far field
}

Minggang Luo, Jian Dong, Junming Zhao, Linhua Liu, Mauro Antezza

\section{To cite this version:}

Minggang Luo, Jian Dong, Junming Zhao, Linhua Liu, Mauro Antezza. Radiative heat transfer between metallic nanoparticle clusters in both near field and far field. Physical Review B, 2019, 99, pp.134207. 10.1103/PhysRevB.99.134207 . hal-02111478

\section{HAL Id: hal-02111478 \\ https://hal.science/hal-02111478}

Submitted on 14 Oct 2020

HAL is a multi-disciplinary open access archive for the deposit and dissemination of scientific research documents, whether they are published or not. The documents may come from teaching and research institutions in France or abroad, or from public or private research centers.
L'archive ouverte pluridisciplinaire $\mathbf{H A L}$, est destinée au dépôt et à la diffusion de documents scientifiques de niveau recherche, publiés ou non, émanant des établissements d'enseignement et de recherche français ou étrangers, des laboratoires publics ou privés. 


\title{
Radiative heat transfer between metallic nanoparticle clusters in both near field and far field
}

\author{
Minggang Luo, ${ }^{1}$ Jian Dong, ${ }^{2}$ Junming Zhao, ${ }^{1, *}$ Linhua Liu, ${ }^{1,2}$ and Mauro Antezza ${ }^{3,4}$ \\ ${ }^{1}$ School of Energy Science and Engineering, Harbin Institute of Technology, 92 West Street, Harbin 150001, China \\ ${ }^{2}$ School of Energy and Power Engineering, Shandong University, Qingdao 266237, China \\ ${ }^{3}$ Laboratoire Charles Coulomb (L2C) UMR 5221 CNRS-Université de Montpellier, F- 34095 Montpellier, France \\ ${ }^{4}$ Institut Universitaire de France, 1 rue Descartes, F-75231 Paris Cedex 05, France
}

(Received 26 January 2019; revised manuscript received 20 March 2019; published 25 April 2019)

\begin{abstract}
Radiative heat transfer (RHT) between two metallic nanoparticles clusters in both near field and far field are explored using many-body radiative heat transfer theory implemented with the coupled electric and magnetic dipole (CEMD) approach, which effectively takes into account the contribution of magnetic polarization of metallic nanoparticles on heat exchange. The effects of magnetic polarization, many-body interaction (MBI), fractal dimension, and relative orientation of the clusters on RHT were analyzed. The results show that the contribution of magnetically polarized eddy-current Joule dissipation dominates the RHT between Ag nanoparticle clusters. If the electric polarization (EP approach) only is considered, the heat conductance will be underestimated as compared with the CEMD approach in both near field and far field regime. The effect of MBI on the RHT between Ag nanoparticle clusters is insignificant at room temperature, which is quite different from the $\mathrm{SiC}$ nanoparticle clusters. For the latter, MBI tends to suppress RHT significantly. The relative orientation has remarkable effect on radiative heat flux for clusters with lacy structure when the separation distance is in the near field. While for the separation distance in far field, both the relative orientation and the fractal dimension has a weak influence on radiative heat flux. This work will help the understanding of thermal transport in dense particulate system.
\end{abstract}

DOI: 10.1103/PhysRevB.99.134207

\section{INTRODUCTION}

Due to rich physics and wide range of potential applications, particularly with the advancement of micro-nano technologies, near-field radiative heat transfer (NFRHT) has received considerable attention in recent years. The fluctuational electrodynamics theory developed by Rytov et al. [1] was well recognized as a theoretical framework to predict NFRHT [2-8], which has been verified by many recent experimental observations [9-17]. In a dense particulate system, the particle separation distance may be comparable to or less than the characteristic thermal wavelength, hence the near-field effect will be significant and become the key factor to influence the thermal radiation transfer characteristics.

Early studies on NFRHT mostly considered system consisting of two bodies, e.g., two plates, two particles, etc. Domingues et al. [18] investigated radiative thermal conductance in the near field by means of molecular dynamics coupled with fluctuation dissipation theorem. Narayanaswamy et al. [19] studied the NFRHT between two spherical particles of arbitrary radius based on a rigorous solution of the fluctuational electrodynamics theory with a quasianalytical approach using vector spherical harmonics expansion. Czapla et al. [20] extended the method developed by Narayanaswamy et al. [19] to investigate NFRHT between two coated spheres with an arbitrary numbers of coatings. Messina et al. [21-23] proposed a scattering operator method to investigate NFRHT

*Corresponding author: jmzhao@hit.edu.cn between two particles of arbitrary shape. Under dipole approximation, Chapuis et al. [24] took into consideration the contribution of magnetic-magnetic polarized eddy-current Joule dissipation (MM contribution) when investigating RHT between two particles. They showed that the electric-electric polarized displacement current dissipation (EE contribution) dominates the RHT between dielectric particles and the MM contribution dominates the RHT between metallic particles. Manjavacas et al. [25] considered the contribution of electromagnetic cross terms, e.g., magnetic-electric polarized eddycurrent Joule dissipation contribution (ME contribution) and electric-magnetic polarized displacement current dissipation (EM contribution) in calculating radiative heat flux between two spherical particles. For dimers consisting of two dielectric particles or two metallic particles, their research results were consistent with the work by Chapuis et al. [24].

For NFRHT in a system consisting of many particles, some important progresses were reported only recently. There are very complex near-field mutual interactions among particles and the approach to deal with NFRHT in two-body system cannot be directly applied to the system of many particles. Ben-Abdallah et al. [26] developed a many-body radiative heat transfer theory to investigate RHT in a many-particle system and the effect of the many-body interaction (MBI) on RHT. Though the theory is based on dipole approximation, this approach is very general and can be effectively applied to predict NFRHT in a system of small particles of any shape, which allows detailed analysis of MBI in a particulate system. They showed that radiative heat flux between two $\mathrm{SiC}$ particles can be enhanced significantly due to MBI after 
the insertion of a third particle [26]. The heat super-diffusion behavior induced by MBI in networks of spherical particles was also predicted [27]. It was also demonstrated that the spatial distribution of particles in a system of particles plays a key role in determining radiative heat flux [28]. In contrast to the enhancement effect of MBI on radiative heat flux observed in the system of three $\mathrm{SiC}$ particles, it was also reported that MBI inhibits the radiative heat flux in dielectric clusters of many particles [29].

Recently, there were some notable theoretical development to deal with NFRHT in system of particles. Krüger et al. [30] proposed trace formulas and applied them to investigate RHT in a many-particle system composed of particles with arbitrary shape and radius. Müller et al. [31] extended the trace formulas to the many-particle system embedded in a nonabsorbing medium. Zhu et al. [32] investigated RHT in a many-particle system without the constraint of reciprocity by means of the trace formulas. Czapla et al. [33] derived formulas for NFRHT in a chain of spheres of arbitrary size, spacing, and isotropic optical properties based on the theoretical frame developed by Narayanaswamy et al. [19], which was validated by the thermal discrete dipole approximation [34] and fluctuating surface currents /boundary element methods) [35]. Becerril et al. [36] investigated near-field energy transfer between three nanoparticles system modulated by coupled multipolar modes and found that coupled modes between nanoparticles provide more channels for NFRHT. By noticing the manybody radiative heat transfer theories did not include the mutual interactions of the electric and magnetic dipole moments, and that most of the studies considered dielectric particles with magnetic dipole moment neglected, Dong et al. [37] developed a coupled electric and magnetic dipole (CEMD) approach for the RHT in a collection of objects in mutual interaction, as an extension of the work of Ben-Abdallah et al. [26]. The CEMD approach takes all the EE, EM, ME, and MM contributions to RHT into consideration, allows the analysis of NFRHT and the effect of MBI in systems containing groups of metallic particles, where the magnetic terms may play an important role. Chen et al. [38] applied the CEMD approach to investigate RHT between two assembled systems of core-shell nanoparticles and observed a similar inhibitive effect of MBI on total radiative heat flux as reported for dielectric particles by Dong et al. [29]. Previous studies have shown that the effect of MBI on RHT is complex in a system of particles and significantly influences the radiative heat flux. It remains unclear about the effect of MBI on the RHT characteristics in system of metallic particles.

In this work, the RHT between two metallic nanoparticles clusters are explored using many-body radiative heat transfer theory with the CEMD approach, which effectively takes into account the contribution of magnetic response of metallic nanoparticles on heat exchange. The effect of magnetic polarization and many-body interaction on NFRHT in a dense particulate system are analyzed as the focus. The effects of fractal dimension and relative orientation of the clusters on NFRHT are also analyzed. This work is organized as follows. In Sec. II, the physical model of the fractal cluster and theoretical aspects of the CEMD approach are presented. The formulas to evaluate the effect of MBI on radiative heat exchange in two nanoparticles clusters are given. In Sec. III, the mechanism of RHT between metallic nanoparticle clusters, the effects of MBI, fractal dimension, and relative orientation of clusters on RHT are analyzed.

\section{MODEL AND METHOD}

\section{A. Nanoparticle cluster generation}

RHT between two metallic nanoparticles clusters is considered. The nanoparticle cluster is described by the following typical statistical rule [39]:

$$
N_{S}=k_{0}\left(\frac{R_{g}}{a}\right)^{D_{f}},
$$

where $N_{S}$ is the number of monomers in the cluster, $D_{f}$ is the fractal dimension, $k_{0}$ is the prefactor, $a$ is the radius of the monomers, and $R_{g}$ is the radius of gyration. The $D_{f}$ is the main factor that describes the compactness of the aggregate. Clusters with three different $D_{f}(1.8,2.3$, and 2.8 ) are generated by the open source program provided by Skorupski et al. [40], shown in Figs. 1(a)-1(c). The number of realizations of clusters has been checked. For more details about the cluster generation, please refer to the previous work [29].The number of monomers in the aggregate $\left(N_{S}\right)$ is set as 400. RHT between two identical absorbing and emitting clusters, of which temperatures are fixed at $T$ and $T+\delta T$, is investigated. The separating gap $(d)$ between clusters is defined as the distance between the bottom of the upper cluster and the top of the lower cluster, shown in Fig. 1(d). Both the separation distance $d$ between clusters and the edge to edge distance between monomers inside the clusters were kept larger than $2 a$, which was taken to ensure the validity of dipolar approximation [26,37].

\section{B. Polarizability of nanoparticle}

In this work, $\mathrm{Ag}$ nanoparticle clusters are used for the calculations. SI unit system is used for all the formulation. For isotropic spherical particles, the electric dipole moment $\mathbf{p}$ and magnetic dipole moment $\mathbf{m}$ induced by the incident electromagnetic field in the vacuum read

$$
\begin{aligned}
& \mathbf{p}=\varepsilon_{0} \alpha_{E} \mathbf{E}^{i n c}, \\
& \mathbf{m}=\alpha_{H} \mathbf{H}^{i n c},
\end{aligned}
$$

where $\alpha_{E}$ and $\alpha_{H}$ are electric and magnetic polarizability, $\mathbf{E}^{i n c}$ and $\mathbf{H}^{i n c}=\mathbf{B}^{i n c} / \mu_{0}$ are the incident electric and magnetic fields, and $\varepsilon_{0}$ is the vacuum dielectric permittivity. The electric and magnetic polarizabilities of a spherical Ag nanoparticle with radius of $5 \mathrm{~nm}$ are shown in Fig. 2. For isotropic spherical particles, the electric and magnetic polarizabilities, $\alpha_{E}$ and $\alpha_{H}$, can be obtained from the first order Lorenz-Mie scattering coefficients as $[41,42]$

$$
\begin{aligned}
& \alpha_{E}=\frac{i 6 \pi}{k^{3}} a_{1}, \\
& \alpha_{H}=\frac{i 6 \pi}{k^{3}} b_{1},
\end{aligned}
$$




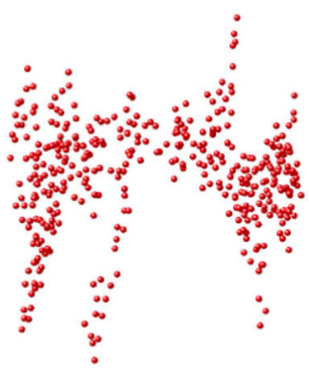

(a) $D_{f}=1.8$

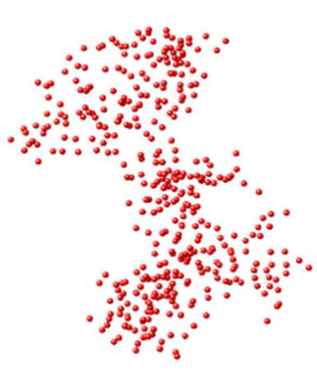

(b) $D_{f}=2.3$

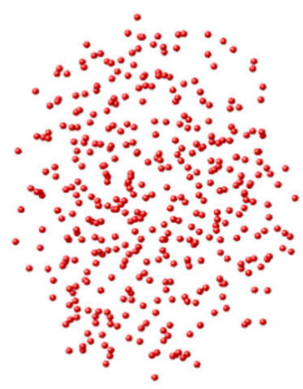

(c) $D_{f}=2.8$

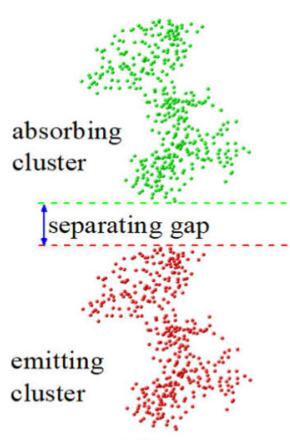

(d)

FIG. 1. Cluster configuration for three different fractal dimensions, $N_{S}$ is 400 and the radius of the monomer is $5 \mathrm{~nm}$. (a) $D_{f}=1.8$, (b) $D_{f}=2.3$, (c) $D_{f}=2.8$ (d) schematic on the definition of the separating gap between the absorbing and emitting clusters.

where $a_{1}$ and $b_{1}$ are the first-order Lorenz-Mie scattering coefficients. The first-order Lorenz-Mie scattering coefficients are calculated from

$$
\begin{aligned}
a_{1} & =\frac{\varepsilon j_{1}(y)\left[x j_{1}(x)\right]^{\prime}-j_{1}(x)\left[y j_{1}(y)\right]^{\prime}}{\varepsilon j_{1}(y)\left[x h_{1}^{(1)}(x)\right]^{\prime}-h_{1}^{(1)}(x)\left[y j_{1}(y)\right]^{\prime}}, \\
b_{1} & =\frac{j_{1}(y)\left[x j_{1}(x)\right]^{\prime}-j_{1}(x)\left[y j_{1}(y)\right]^{\prime}}{j_{1}(y)\left[x h_{1}^{(1)}(x)\right]^{\prime}-h_{1}^{(1)}(x)\left[y j_{1}(y)\right]^{\prime}},
\end{aligned}
$$

where $x=k R, y=\sqrt{\varepsilon} k R, k$ is wave vector, $R$ is the particle radius, $\varepsilon$ is the dielectric permittivity, $j_{1}(x)=\sin (x) / x^{2}-$ $\cos (x) / x$ and $h_{1}^{(1)}(x)=e^{i x}\left(1 / i x^{2}-1 / x\right)$ are Bessel functions and spherical Hankel functions, respectively. The dielectric permittivity of $\mathrm{Ag}$ is described by the Drude model $[29,43]$

$$
\varepsilon(\omega)=1-\frac{\omega_{p}^{2}}{\omega^{2}+i \gamma \omega},
$$

where $\omega$ is angular frequency, $\omega_{p}$ is $1.37 \times 10^{16} \mathrm{rad} \mathrm{s}^{-1}$ and $\gamma$ is $2.73 \times 10^{13} \mathrm{rad} \mathrm{s}^{-1}$.

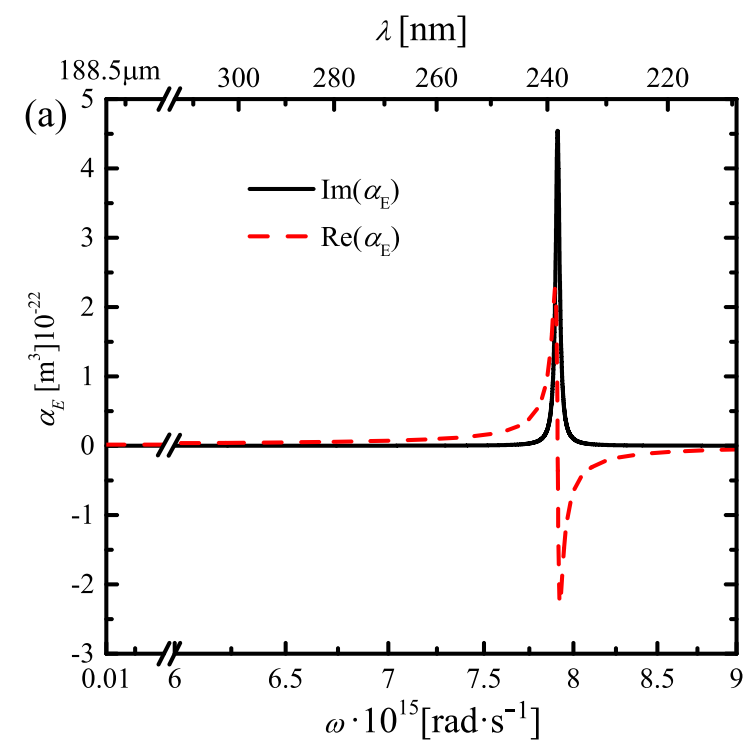

Localized surface plasmon resonance of the Ag nanoparticle lies in the optical frequency range, as shown in Fig. 2(a), which cannot be excited thermally. Hence, RHT between Ag nanoparticles cannot be as strong as the RHT between $\mathrm{SiC}$ dielectric particles, which can support low frequency localized surface phonon resonance. However, the magnetic response of the Ag nanoparticle is strong in the long wavelength range located near the thermal wavelength [Fig. 2(b)], indicating significant contribution of magnetic response to RHT in the particulate system.

\section{Theoretical aspect}

According to the Poynting theorem, the power dissipation induced by the incident electromagnetic wave is [24]

$$
P=\left\langle\frac{\partial \mathbf{p}}{\partial t} \cdot \mathbf{E}^{i n c}-\mathbf{m} \cdot \frac{\partial \mathbf{B}^{i n c}}{\partial t}\right\rangle,
$$

where $\langle\cdot\rangle$ means ensemble average, $P$ is the power dissipation, and $t$ is time. The first term in the right hand side of Eq. (9) is the electric polarized displacement current

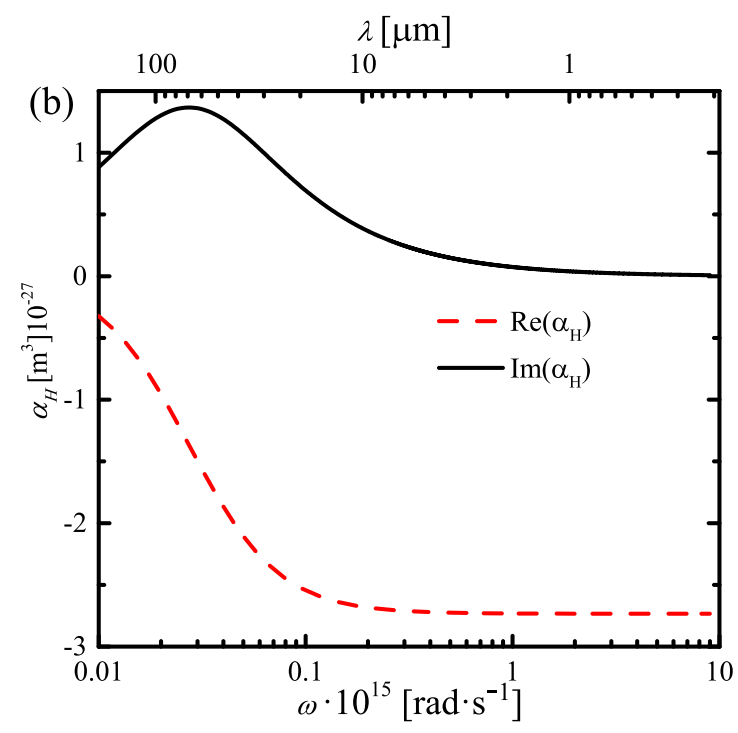

FIG. 2. The electric polarizability $\alpha_{E}$ (a) and the magnetic polarizability $\alpha_{H}$ (b) of the Ag nanoparticle, which are calculated from Eqs. (4) and (5) using the first-order Lorenz-Mie scattering coefficients. The radius of the nanoparticle is $5 \mathrm{~nm}$. 
dissipation and the second term is the magnetic polarized eddy-current Joule dissipation. The corresponding crossspectral density $P_{\omega}$ is

$$
P_{\omega}=\omega \operatorname{Im}\left\langle\mathbf{p} \cdot \mathbf{E}^{i n c *}+\mathbf{m} \cdot \mathbf{B}^{i n c *}\right\rangle
$$

where the superscript $*$ denotes the conjugation of the corresponding complex vector. For metallic nanoparticle, in addition to the electric contribution, the magnetic contribution to the power dissipation will be significant and even become dominant. In this work, the CEMD approach [37] is used to calculate RHT between metallic nanoparticle clusters, which effectively takes into consideration of the EE, EM, ME, and MM contributions to RHT and hence allows the analysis of NFRHT in a system of metallic particles. In free space, the electromagnetic field at the field point induced by an electric dipole $\mathbf{p}$ at source point is

$$
\mathbf{E}_{E E}=\mu_{0} \omega^{2} G_{0}^{E E} \mathbf{p}, \quad \mathbf{H}_{M E}=k \omega G_{0}^{M E} \mathbf{p}
$$

where $\mu_{0}$ is the vacuum permeability, $G_{0}^{E E}$ and $G_{0}^{M E}$ are Green's functions in free space,

$$
\begin{gathered}
G_{0}^{E E}=\frac{e^{i k r}}{4 \pi r}\left[\left(1+\frac{i k r-1}{k^{2} r^{2}}\right) \mathbb{I}_{3}+\frac{3-3 i k r-k^{2} r^{2}}{k^{2} r^{2}} \hat{\mathbf{r}} \otimes \hat{\mathbf{r}}\right] \\
G_{0}^{M E}=\frac{e^{i k r}}{4 \pi r}\left(1-\frac{1}{i k r}\right)\left[\begin{array}{ccc}
0 & -\hat{r}_{z} & \hat{r}_{y} \\
\hat{r}_{z} & 0 & -\hat{r}_{x} \\
-\hat{r}_{y} & \hat{r}_{x} & 0
\end{array}\right],
\end{gathered}
$$

where $\mathbb{I}_{3}$ is a $3 \times 3$ identity matrix, $r$ is the magnitude of the separation vector $\mathbf{r}=\mathbf{r}_{f}-\mathbf{r}_{s}$ between the source point $\mathbf{r}_{s}$ and field point $\mathbf{r}_{f}, \hat{\mathbf{r}}$ is the unit vector $\mathbf{r} / r$ and $\hat{r}_{v=x, y, z}$ denotes its three Cartesian components, $\otimes$ denotes the outer product of vectors. The electromagnetic field at $\mathbf{r}_{f}$ induced by the magnetic dipole $\mathbf{m}$ at $\mathbf{r}_{s}$ is

$$
\mathbf{E}_{E M}=\mu_{0} \omega k G_{0}^{E M} \mathbf{m}, \quad \mathbf{H}_{M M}=k^{2} G_{0}^{M M} \mathbf{m},
$$

where $G_{0}^{E M}=-G_{0}^{M E}$ and $G_{0}^{M M}=G_{0}^{E E}$ are related Green's functions in free space. In a many-particle system, the Green's functions link the $j t h$ electromagnetic dipoles and their induced electromagnetic field at ith particle as

$$
\begin{gathered}
\mathbf{E}_{j \rightarrow i, E E}=\mu_{0} \omega^{2} G_{i j}^{E E} \mathbf{p}_{j}, \quad \mathbf{H}_{j \rightarrow i, M E}=k \omega G_{i j}^{M E} \mathbf{p}_{j}, \\
\mathbf{E}_{j \rightarrow i, E M}=\mu_{0} \omega k G_{i j}^{E M} \mathbf{m}_{j}, \quad \mathbf{H}_{j \rightarrow i, M M}=k^{2} G_{i j}^{M M} \mathbf{m}_{j},
\end{gathered}
$$

where $G_{i j}^{E E}, G_{i j}^{M E}, G_{i j}^{E M}$, and $G_{i j}^{M M}$ are the Green's functions in the many-particle system, which can be deduced from the Green's functions in free space as follows:

$$
\left(\begin{array}{cccc}
0 & \mathbb{G}_{12} & \cdots & \mathbb{G}_{1 \mathrm{~N}} \\
\mathbb{G}_{21} & 0 & \ddots & \vdots \\
\vdots & \vdots & \ddots & \mathbb{G}_{(\mathrm{N}-1) \mathrm{N}} \\
\mathbb{G}_{\mathrm{N} 1} & \mathbb{G}_{\mathrm{N} 2} & \cdots & 0
\end{array}\right)=\left(\begin{array}{cccc}
0 & \mathbb{G}_{0,12} & \cdots & \mathbb{G}_{0,1 \mathrm{~N}} \\
\mathbb{G}_{0,21} & 0 & \ddots & \vdots \\
\vdots & \vdots & \ddots & \mathbb{G}_{0,(\mathrm{~N}-1) \mathrm{N}} \\
\mathbb{G}_{0, \mathrm{~N} 1} & \mathbb{G}_{0, \mathrm{~N} 2} & \cdots & 0
\end{array}\right) \mathbf{A}^{-1}
$$

in which the elements in the matrix are given as

$$
\mathbb{G}_{i j}=\left[\begin{array}{cc}
\mu_{0} \omega^{2} G_{i j}^{E E} & \mu_{0} \omega k G_{i j}^{E M} \\
k \omega G_{i j}^{M E} & k^{2} G_{i j}^{M M}
\end{array}\right], \quad \mathbb{G}_{0, i j}=\left[\begin{array}{cc}
\mu_{0} \omega^{2} G_{0, i j}^{E E} & \mu_{0} \omega k G_{0, i j}^{E M} \\
k \omega G_{0, i j}^{M E} & k^{2} G_{0, i j}^{M M}
\end{array}\right],
$$

and $\boldsymbol{A}$ is a matrix including the many-body interaction defined as

$$
\mathbf{A}=\mathbb{I}_{6 \mathrm{~N}}-\left[\begin{array}{cccc}
0 & \alpha_{1} \mathbb{G}_{0,12} & \cdots & \alpha_{1} \mathbb{G}_{0,1 \mathrm{~N}} \\
\alpha_{2} \mathbb{G}_{0,21} & 0 & \ddots & \vdots \\
\vdots & \vdots & \ddots & \alpha_{\mathrm{N}-1} \mathbb{G}_{0,(\mathrm{~N}-1) \mathrm{N}} \\
\alpha_{\mathrm{N}} \mathbb{G}_{0, \mathrm{~N} 1} & \cdots & \alpha_{\mathrm{N}} \mathbb{G}_{0, \mathrm{~N}(\mathrm{~N}-1)} & 0
\end{array}\right], \quad \alpha_{i}=\left[\begin{array}{cc}
\varepsilon_{0} \alpha_{E}^{i} \mathbb{I}_{3} & 0 \\
0 & \alpha_{H}^{i} \mathbb{I}_{3}
\end{array}\right],
$$

where $\alpha_{i}$ is a $6 \times 6$ matrix, $\mathbb{I}_{6 \mathrm{~N}}$ is a $6 \mathrm{~N} \times 6 \mathrm{~N}$ identity matrix. The power absorbed by the ith particle excited with thermal emission from the $j t h$ particle is

$$
\begin{aligned}
P_{j \rightarrow i}= & 2 \int_{0}^{+\infty} \frac{d \omega}{2 \pi} \omega\left[\operatorname{Im}\left(\chi_{E}^{i}\right) \varepsilon_{0}\left\langle\mathbf{E}_{j \rightarrow i}^{i n c} \cdot \mathbf{E}_{j \rightarrow i}^{i n c *}\right\rangle\right. \\
& \left.+\operatorname{Im}\left(\chi_{H}^{i}\right) \mu_{0}\left\langle\mathbf{H}_{j \rightarrow i}^{i n c} \cdot \mathbf{H}_{j \rightarrow i}^{i n c *}\right)\right],
\end{aligned}
$$

where $\chi_{E}=\alpha_{E}-\frac{i k^{3}}{6 \pi}\left|\alpha_{E}\right|^{2}$ and $\chi_{H}=\alpha_{H}-\frac{i k^{3}}{6 \pi}\left|\alpha_{H}\right|^{2}$. The incident electromagnetic fields are excited by the thermal fluctuating electric and magnetic dipole moment $\mathbf{p}_{j}^{\text {fluc }}$ and $\mathbf{m}_{j}^{\text {fluc }}$, expressed as

$$
\begin{gathered}
\mathbf{E}_{j \rightarrow i}^{i n c}=\mu_{0} \omega^{2} G_{i j}^{E E} \mathbf{p}_{j}^{f l u c}+\mu_{0} \omega k G_{i j}^{E M} \mathbf{m}_{j}^{f l u c} \\
\mathbf{H}_{j \rightarrow i}^{i n c}=k \omega G_{i j}^{M E} \mathbf{p}_{j}^{f l u c}+k^{2} G_{i j}^{M M} \mathbf{m}_{j}^{f l u c}
\end{gathered}
$$


With the application of the fluctuation dissipation theorem for electric and magnetic dipole moment [19,34],

$$
\begin{aligned}
\left\langle p_{j, \alpha}^{f l u c} p_{j, \beta}^{f l u c^{*}}\right\rangle & =2 \frac{\varepsilon_{0}}{\omega} \operatorname{Im}\left(\chi_{E}^{j}\right) \Theta\left(\omega, T_{j}\right) \delta_{\alpha \beta}, \\
\left\langle m_{j, \alpha}^{f l u c} m_{j, \beta}^{f l u c^{*}}\right\rangle & =\frac{2}{\omega \mu_{0}} \operatorname{Im}\left(\chi_{H}^{j}\right) \Theta\left(\omega, T_{j}\right) \delta_{\alpha \beta},
\end{aligned}
$$

the power absorbed by the ith particle caused by the $j t h$ particle can be written in Landauer-like formalism as

$$
P_{j \rightarrow i}=3 \int_{0}^{+\infty} \frac{d \omega}{2 \pi} \Theta\left(\omega, T_{j}\right) \mathcal{T}_{i, j}(\omega)
$$

where $\mathcal{T}_{i, j}(\omega)$ is the transmission coefficient from the $j t h$ particle to the ith particle given as

$$
\begin{aligned}
\mathcal{T}_{i, j}(\omega)= & \frac{4}{3} k^{4}\left[\operatorname{Im}\left(\chi_{E}^{i}\right) \operatorname{Im}\left(\chi_{E}^{j}\right) \operatorname{Tr}\left(G_{i j}^{E E} G_{i j}^{E E \dagger}\right)\right. \\
& +\operatorname{Im}\left(\chi_{E}^{i}\right) \operatorname{Im}\left(\chi_{H}^{j}\right) \operatorname{Tr}\left(G_{i j}^{E M} G_{i j}^{E M \dagger}\right) \\
& +\operatorname{Im}\left(\chi_{H}^{i}\right) \operatorname{Im}\left(\chi_{E}^{j}\right) \operatorname{Tr}\left(G_{i j}^{M E} G_{i j}^{M E^{\dagger}}\right) \\
& \left.+\operatorname{Im}\left(\chi_{H}^{i}\right) \operatorname{Im}\left(\chi_{H}^{j}\right) \operatorname{Tr}\left(G_{i j}^{M M} G_{i j}^{M M^{\dagger}}\right)\right],
\end{aligned}
$$

where the four terms in the bracket are corresponding to the EE, EM, ME, and MM contributions to the exchanged radiative power, respectively. The radiative power exchanged between particle $i$ and $j$ in the many-particle system can be calculated from

$$
\begin{aligned}
P_{j \leftrightarrow i} & =P_{j \rightarrow i}-P_{i \rightarrow j} \\
& =3 \int_{0}^{+\infty} \frac{d \omega}{2 \pi}\left(\Theta\left(\omega, T_{j}\right)-\Theta\left(\omega, T_{i}\right)\right) \mathcal{T}_{i, j}(\omega) .
\end{aligned}
$$

The net exchanged RHT power between two nanoparticle clusters considering MBI obtained from CEMD is calculated from

$$
\varphi=\sum_{j=1}^{N e} \sum_{i=1}^{N a} P_{j \leftrightarrow i},
$$

where $\mathrm{Ne}$ is the number of particles in the emitting cluster, and $N a$ is the number of particles in the absorbing cluster. The thermal conductance $(G)$ between the two nanoparticle clusters is defined as

$$
G=\lim _{\delta T \rightarrow 0} \frac{\varphi}{\delta T},
$$

where $\delta T$ is the temperature difference between the emitting cluster and the absorbing cluster. When MBI is not considered, namely, the existence of all other particles does not change the system Green function, hence the system Green function is just the Green function in vacuum, and the transmission coefficient between particle $i$ and $j$ is calculated from

$$
\begin{aligned}
\mathcal{T}_{i, j}^{0}(\omega)= & \frac{4}{3} k^{4}\left[\operatorname{Im}\left(\chi_{E}^{i}\right) \operatorname{Im}\left(\chi_{E}^{j}\right) \operatorname{Tr}\left(G_{0, i j}^{E E} G_{0, i j}^{E E \dagger}\right)\right. \\
& +\operatorname{Im}\left(\chi_{E}^{i}\right) \operatorname{Im}\left(\chi_{H}^{j}\right) \operatorname{Tr}\left(G_{0, i j}^{E M} G_{0, i j}^{E M \dagger}\right) \\
& +\operatorname{Im}\left(\chi_{H}^{i}\right) \operatorname{Im}\left(\chi_{E}^{j}\right) \operatorname{Tr}\left(G_{0, i j}^{M E} G_{0, i j}^{M E \dagger}\right) \\
& \left.+\operatorname{Im}\left(\chi_{H}^{i}\right) \operatorname{Im}\left(\chi_{H}^{j}\right) \operatorname{Tr}\left(G_{0, i j}^{M M} G_{0, i j}^{M M \dagger}\right)\right] .
\end{aligned}
$$

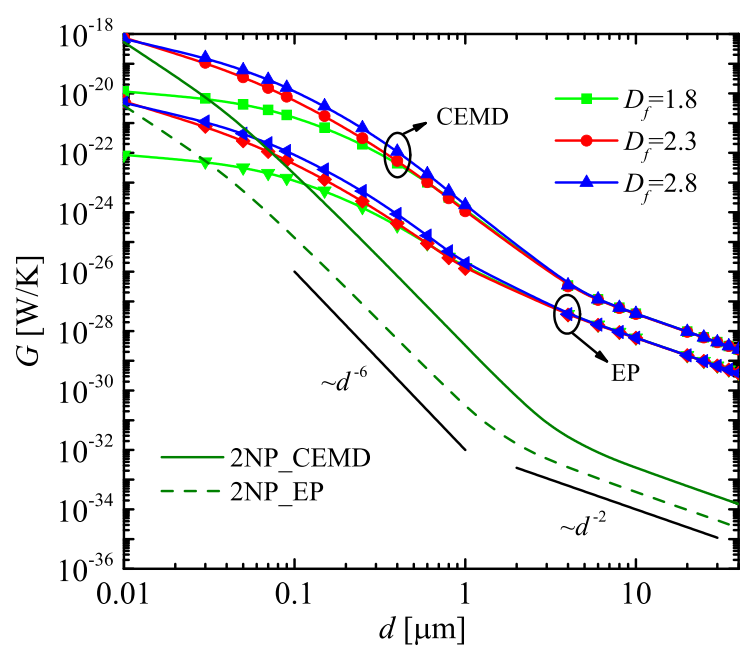

FIG. 3. Thermal conductance between two Ag nanoparticles clusters at various fractal dimensions. Both the CEMD and EP approach are used to calculate thermal conductance.

Then by omitting the MBI, the RHT power exchanged between two particles $\left(P_{j \leftrightarrow i}^{0}\right)$, the net exchanged RHT power between two clusters $\left(\varphi_{0}\right)$, and the thermal conductance without MBI $\left(G_{0}\right)$ can be calculated using Eqs. (27)-(29) with $\mathcal{T}_{i, j}^{0}(\omega)$, respectively. Note that this definition of the RHT without MBI is consistent with the previous definition by Dong et al. [29], which directly calculates RHT between two particles.

The thermal conductance calculated considering only the EE contribution $G_{E E}$, namely, only using the first term in the transmission coefficient in Eq. (26), is the same as the approach of the original many-body radiative heat transfer theory [26], denoted as the EP approach in the following for comparison.

\section{RESULTS AND DISCUSSION}

Radiative heat transfer between two Ag nanoparticles clusters was investigated at various fractal dimensions $\left(D_{f}\right)$ and separating gaps $(d)$. The thermal conductance was calculated at $300 \mathrm{~K}$ for all cases. The total thermal conductance was integrated over an angular frequency range from $0.1 \times$ $10^{14} \mathrm{rad} \mathrm{s}^{-1}$ to $90 \times 10^{14} \mathrm{rad} \mathrm{s}^{-1}$. A proper frequency resolution has been used to integrate spectral thermal conductance to obtain an accurate thermal conductance using the composite Simpson numerical integration method. The calculation time for one case (one data point in Fig. 3) in this work took about $12 \mathrm{~h}$ on a work station with an Intel Xeon(R) CPU E5-2690, $2.60 \mathrm{GHz}$, and main memory of $128 \mathrm{~GB}$.

\section{A. RHT mechanism between metallic nanoparticle clusters}

Thermal conductance between two Ag nanoparticles clusters as a function of separating gap $(d)$ is shown in Fig. 3. Both $G$ and $G_{E E}$ are shown and the lines of $1 / d^{6}$ and $1 / d^{2}$ are added as a reference. As shown, when $d$ is small (less than $1 \mu \mathrm{m}$ ), thermal conductance between two Ag nanoparticles clusters increases with the $D_{f}$. While $d$ is large enough (larger than $1 \mu \mathrm{m}), D_{f}$ has little effect on the thermal conductance. The 


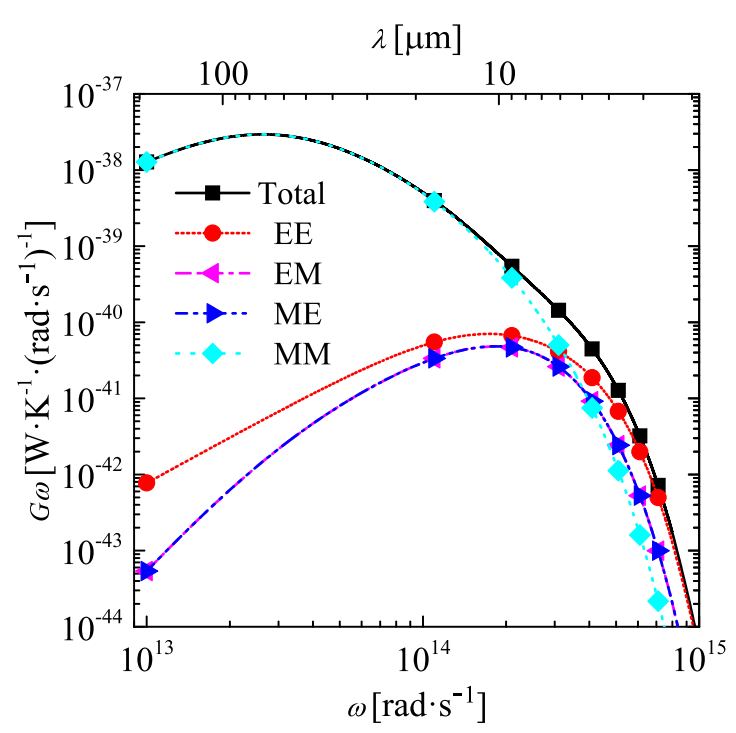

FIG. 4. Spectral thermal conductance, $G_{\omega}$, due to EE, EM, ME and MM contribution, respectively. $D_{f}$ of the cluster is set as 2.8 and the separation gap between clusters is $1 \mu \mathrm{m}$.

thermal conductance between two $\mathrm{Ag}$ nanoparticles is even larger than that of two clusters when $d$ is sufficiently small for smaller fractal dimension, e.g., $D_{f}=1.8$. The reason for this phenomenon is that the distance between nanoparticles in proximity has priority over the number of the emitting and absorbing nanoparticles in determining the near-field thermal conductance. The straight-line distance between the two nanoparticles is usually smaller than the closest distance between two nanoparticles from the emitting and absorbing clusters, when the $D_{f}$ is not too large. As the $D_{f}$ increases, the straight-line distance between the two nanoparticles in proximity from the emitting and absorbing clusters approaches that of two nanoparticles, which results in $G$ between clusters is larger than that of two nanoparticles. The number of particles in clusters begins to dominate the near-field thermal conductance as $D_{f}$ of clusters increases to 2.3 and 2.8. Thermal conductance between two nanoparticles decays as $1 / d^{6}$ in near field for $d$ less than $1 \mu \mathrm{m}$ and decays as $1 / d^{2}$ in the far field. However, thermal conductance between two nanoparticle clusters decays slower than $1 / d^{6}$ in near field and decays as $1 / d^{2}$ in the far field. This may be attributed to the MBI in the nanoparticle clusters.

For dielectric nanoparticle, radiative heat transfer is dominated by the electric displacement current dissipation. While for metallic nanoparticle, eddy-current Joule dissipation due to the variation of magnetic field in the particle dominates the radiative heat transfer. Thermal conductance obtained from the EP approach, considering only electric polarization response, is also shown in Fig. 3. For both of the metallic nanoparticle clusters and two metallic nanoparticles, the EP approach underestimates the radiative heat transfer as compare to the CEMD in both near field and far field.

To further explain the above observation on total thermal conductance, the spectral thermal conductance $G_{\omega}$ due to EE, EM, ME, and MM contributions are presented in Fig. 4, where $d$ is $1 \mu \mathrm{m}$ and $D_{f}$ is 2.8 . For metallic nanoparticle clusters, the MM contribution dominates the thermal conductance. $G_{\omega}$ due to the EE contribution is far less than that of the MM contribution, which results in the underestimation of RHT using the EP approach. The peak of the $G_{\omega}$ due to MM contribution locates at $100 \mu \mathrm{m}$, which is consistent with the peak of the imaginary part of the magnetic polarizability of metallic $\mathrm{Ag}$ as shown in Fig. 2(b). However, the peaks of the $G_{\omega}$ due to EE, ME, and EM contributions all locate at about $10 \mu \mathrm{m}$, which corresponds to the characteristic thermal wavelength at $300 \mathrm{~K}$.

\section{B. Effect of many-body interaction}

Previous studies reported that MBI inhibits the RHT in dielectric and core-shell particle clusters [29,38], although it enhances RHT in three SiC particle system [26]. It is still unclear whether MBI inhibits or enhances RHT in metallic nanoparticle clusters. In this section, MBI on RHT in Ag nanoparticle clusters is investigated. To evaluate the MBI on RHT, a definition of the enhancement factor of RHT due to $\mathrm{MBI}$ is given as

$$
E=\frac{\varphi}{\varphi_{0}}
$$

which is defined as the ratio of the net exchanged RHT power between two nanoparticle clusters with considering MBI to that calculated without considering MBI. The enhancement factor can be calculated using the spectrally net exchanged RHT power or the total net exchanged RHT power. In order to understand the MBI on RHT between metallic nanoparticle clusters, it is necessary to investigate the simplest case (a system of two nanoparticles) at first. The enhancement factor between two Ag nanoparticles is shown in Fig. 5. For two nanoparticles separated by $0.01 \mu \mathrm{m}$, the spectrally distributed $E$ is nearly equal to 1 in the infrared frequency, although in the optical frequency range the $E$ is much larger than 1, which means that the MBI has little effect on the thermal radiative heat transfer between two $\mathrm{Ag}$ nanoparticles. The increase in the separation gap between $\mathrm{Ag}$ particles decreases the enhancement factor in the optical frequency range, indicating a weakening of MBI. From the point view of total $E$, the MBI is insignificant for the Ag nanoparticles, which keeps nearly a constant value of 1.0 at different separation distance, shown as the black line in Fig. 5(b). For the SiC nanoparticles, the MBI inhibits the RHT in the near field, while it has little effect on the RHT in the far field, shown as the red line in Fig. 5(b).

The spectral enhancement factor between two Ag nanoparticles clusters at four different separating gaps is shown in Fig. 6(a). The spectral $E$ increases dramatically in the optical frequency around the resonance, which agree with the result of the two nanoparticle system. The spectral $E$ in the infrared frequency approaches 1 , which means that the MBI has little effect on the RHT between Ag nanoparticle clusters. The enhancement factor of RHT for both Ag nanoparticle clusters and dielectric clusters of $\mathrm{SiC}$ is shown as a function of $d$ in Fig. 6(b). For dielectric nanoparticle clusters, the MBI inhibits RHT in both near field and far field. In contrast, generally speaking, the enhancement factor for $\mathrm{Ag}$ nanoparticle clusters keeps nearly a constant value of 1.0 with various $d$, which means that MBI has an insignificant effect on the RHT for metallic nanoparticle clusters in both near field and far field. 


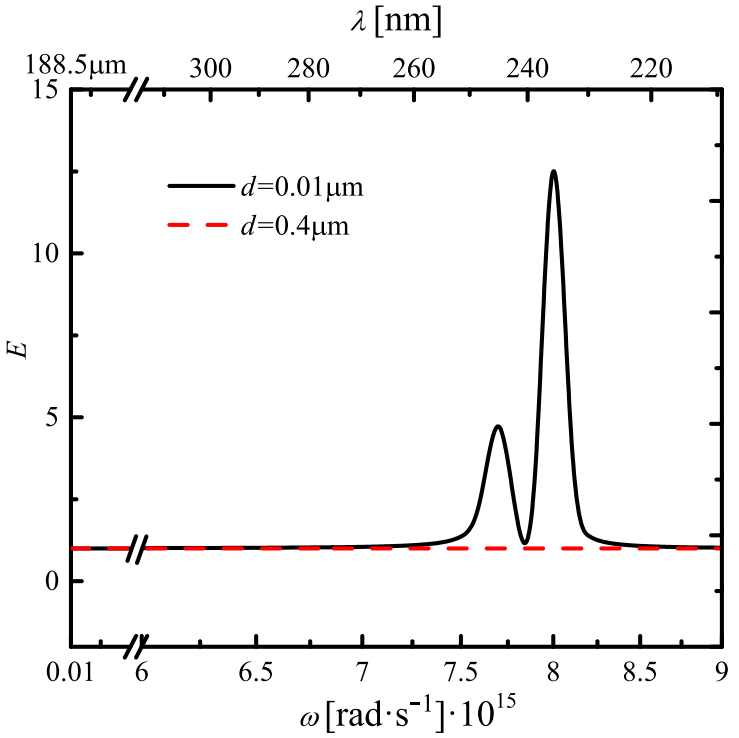

(a)

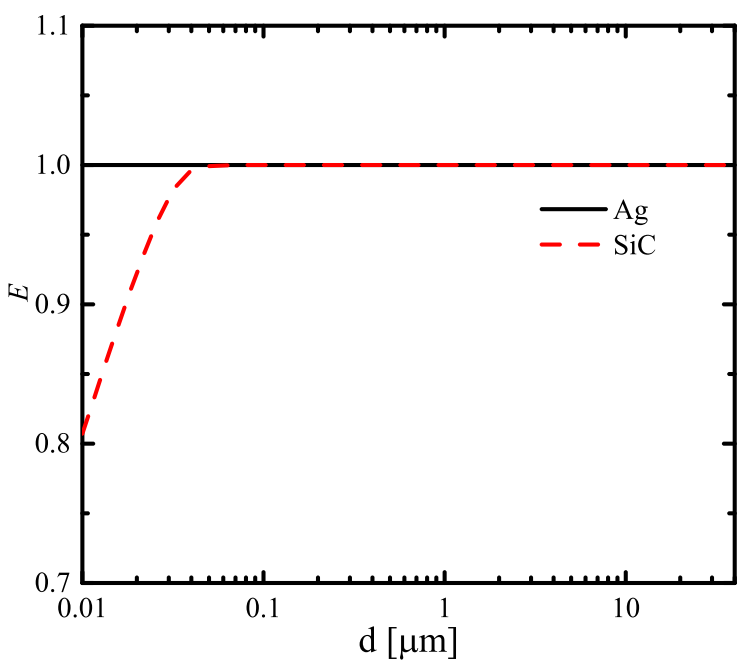

(b)

FIG. 5. Enhancement factor of radiative thermal conductance between two Ag nanoparticles due to MBI: (a) spectrally distributed enhancement factor at two different separating gaps; (b) total enhancement factor as a function of the separating gap, ranging from near field to far field.

Meanwhile, the inhibition of MBI on RHT for dielectric nanoparticle clusters decreases with the increasing separating gap, which is consistent with the results obtained by the EP approach [29].

There is no resonance in the infrared range of polarizability for the metallic nanoparticle as shown in Fig. 2. Hence the plasmonic coupling between neighboring particles is very weak at room temperature, indicating the $\mathrm{MBI}$ in the $\mathrm{Ag}$ clusters is very weak. As such, for Ag clusters with different fractal dimensions, the enhancement factor $E$ due to MBI is about a constant value of 1.0. However, for the $\mathrm{SiC}$ nanopar-

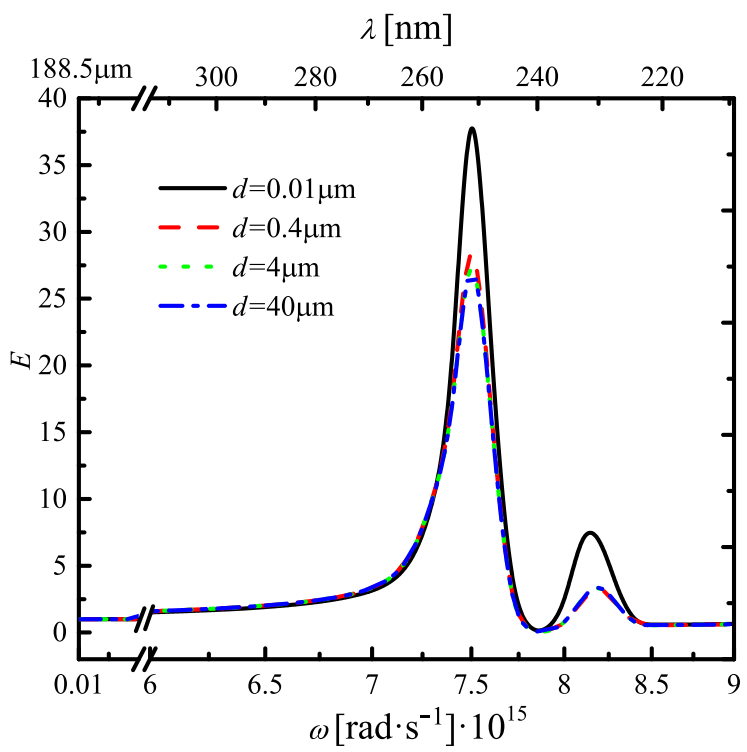

(a) ticle, there is a strong plasmonic resonance at about $10 \mu \mathrm{m}$. Hence the plasmonic coupling between neighboring particles is strong at room temperature, which results in a strong MBI. The strong MBI then induces a significant dependence of $G$ on fractal dimension for $\mathrm{SiC}$ clusters.

\section{Effect of relative orientation}

The thermal conductance between two Ag nanoparticles clusters in three different relative orientations (parallel, oblique, and vertical) is shown as a function of separation

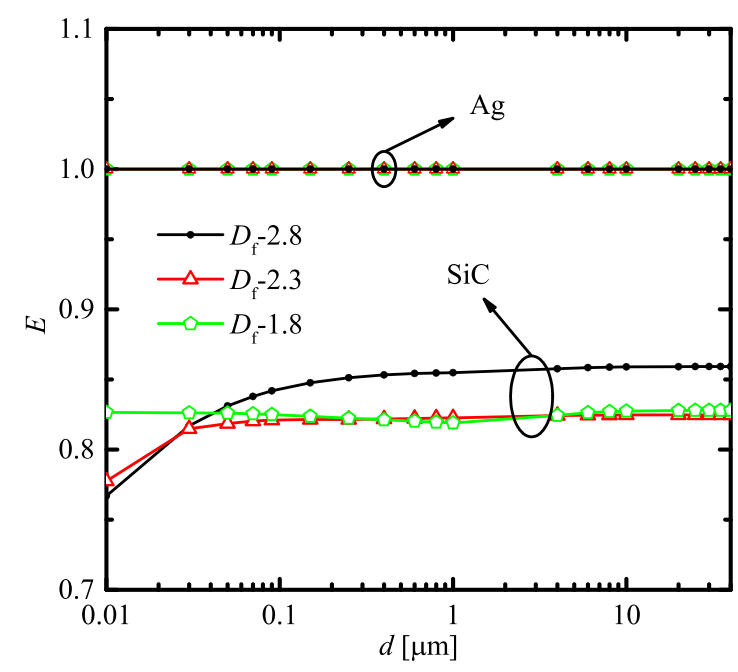

(b)

FIG. 6. (a) Enhancement factor of RHT between two Ag nanoparticle clusterswith $D_{f} 2.3$ for different separation gaps. (b) Enhancement factor of RHT for $\mathrm{SiC}$ and $\mathrm{Ag}$ nanoparticle clusters as a function of the separating gap. 


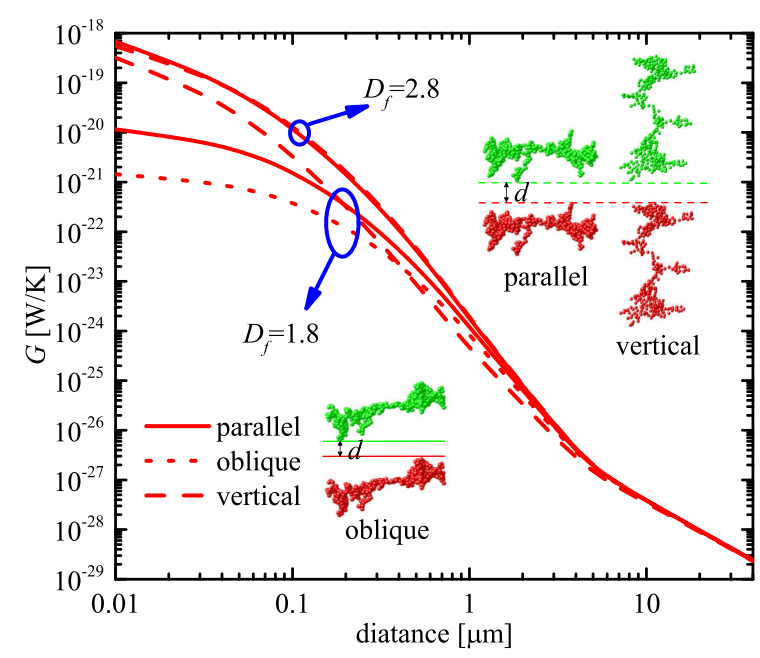

FIG. 7. Thermal conductance between two Ag nanoparticle clusters at different relative orientations.

distance $d$ in Fig. 7. When $d$ is larger than $3 \mu \mathrm{m}$, both relative orientation and fractal dimension have little effect on the RHT. For clusters with high fractal dimension $\left(D_{f}=2.8\right)$, rotation of the clusters has little effect on the RHT. While for clusters with low fractal dimension $\left(D_{f}=1.8\right)$, rotation of the clusters has a significant effect on the RHT in the near field. The nanoparticles from clusters in proximity and the number of nanoparticles in the emitting and the absorbing clusters play a dominant role in determining the NFRHT between nanoparticle clusters. For clusters with $D_{f}$ of 1.8 , the thermal conductance at vertical orientation is much larger than that oblique and parallel orientation. The straight-line distance of particles in proximity in the emitting and absorbing clusters at vertical orientation is much smaller than that at oblique and parallel orientation, which is a reason for the relatively large thermal conductance at the near-field regime. In general, relative orientation has remarkable effect on radiative heat flux for clusters with lacy structure when the separation distance is in the near field. While for the separation distance in the far field, both the relative orientation and the fractal dimension has a weak influence on the radiative heat flux.

\section{CONCLUSIONS}

The near-field effect is a key factor for influencing thermal radiation transfer in dense particulate systems when the particle separation distance is comparable to or less than the characteristic thermal wavelength. Near-field radiative heat transfer between Ag nanoparticle clusters in both the near field and far field was studied by using the CEMD approach, considering contributions of all four electromagnetic field terms from electric and magnetic polarizations, namely EE, EM, ME, and MM. The EP approach that considers only the contribution of electric polarization underestimates the RHT in both the near field and far field, which is attributed to the dominant role of the MM contribution in the RHT between metallic nanoparticle clusters. The effect of MBI on the RHT between Ag nanoparticle clusters is insignificant at room temperature, while MBI significantly inhibits the RHT between dielectric nanoparticle clusters at the nearfield regime. The effect of fractal dimension and relative orientation on RHT are also analyzed. When the separation distance is small (less than $1 \mu \mathrm{m}$ ), the thermal conductance between two Ag nanoparticle clusters increases with the fractal dimension. Whereas when the separation distance is large enough, the fractal dimension shows little effect on the thermal conductance. The relative orientation has a remarkable effect on radiative heat flux for clusters with lacy structure when the separation distance is in the near field. Whereas for the separation distance in the far field, both the relative orientation and the fractal dimension have a weak influence on radiative heat flux. A possible extension of this work is to take the interplay between the periodic configuration of a many-particle system and RHT into consideration [44] in the future.

\section{ACKNOWLEDGMENTS}

The support by the National Natural Science Foundation of China (Grant No. 51336002) and the Fundamental Research Funds for the Central Universities (Grant No. HIT.BRETIII.201415) are gratefully acknowledged.
[1] S. M. Rytov, Y. A. Kravtsov, and V. I. Tatarskii, Priniciples of Statistical Radiophysics (Springer-Verlag, Berlin, 1989), Vol. 3.

[2] D. Polder and M. Van Hove, Phys. Rev. B 4, 3303 (1971).

[3] J. J. Loomis and H. J. Maris, Phys. Rev. B 50, 18517 (1994).

[4] R. Carminati and J.-J. Greffet, Phys. Rev. Lett. 82, 1660 (1999).

[5] A. V. Shchegrov, K. Joulain, R. Carminati, and J.-J. Greffet, Phys. Rev. Lett. 85, 1548 (2000).

[6] A. I. Volokitin and B. N. J. Persson, Phys. Rev. B 63, 205404 (2001).

[7] A. Narayanaswamy and G. Chen, Appl. Phys. Lett. 82, 3544 (2003).

[8] A. I. Volokitin and B. N. J. Persson, Phys. Rev. B 69, 045417 (2004).

[9] S. Shen, A. Narayanaswamy, and G. Chen, Nano Lett. 9, 2909 (2009).
[10] K. Kloppstech, N. Könne, S. A. Biehs, A. W. Rodriguez, L. Worbes, D. Hellmann, and A. Kittel, Nat. Commun. 8 (2017).

[11] E. Rousseau, A. Siria, G. Jourdan, S. Volz, F. Comin, J. Chevrier, and J.-J. Greffet, Nat. Photonics 3, 514 (2009).

[12] M. Ghashami, H. Geng, T. Kim, N. Iacopino, S. K. Cho, and K. Park, Phys. Rev. Lett. 120, 175901 (2018).

[13] R. S. Ottens, V. Quetschke, S. Wise, A. A. Alemi, R. Lundock, G. Mueller, D. H. Reitze, D. B. Tanner, and B. F. Whiting, Phys. Rev. Lett. 107, 014301 (2011).

[14] K. Kim, B. Song, V. Fernández-Hurtado, W. Lee, W. Jeong, L. Cui, D. Thompson, J. Feist, M. T. H. Reid, and F. J. GarcíaVidal, Nature (London) 528, 387 (2015).

[15] R. St-Gelais, B. Guha, L. Zhu, S. Fan, and M. Lipson, Nano Lett. 14, 6971 (2014). 
[16] B. Song, Y. Ganjeh, S. Sadat, D. Thompson, A. Fiorino, J. Feist, F. J. Garciavidal, J. C. Cuevas, P. Reddy, and E. Meyhofer, Nat. Nanotechnol. 10, 253 (2015).

[17] M. Lim, S. S. Lee, and B. J. Lee, Phys. Rev. B 91, 195136 (2015).

[18] G. Domingues, S. Volz, K. Joulain, and J.-J. Greffet, Phys. Rev. Lett. 94, 085901 (2005).

[19] A. Narayanaswamy and G. Chen, Phys. Rev. B 77, 075125 (2008).

[20] B. Czapla and A. Narayanaswamy, Phys. Rev. B 96, 125404 (2017).

[21] R. Messina and M. Antezza, Phys. Rev. A 84, 042102 (2011).

[22] R. Messina and M. Antezza, Europhys. Lett. 95, 61002 (2011).

[23] R. Messina and M. Antezza, Phys. Rev. A 89, 052104 (2014).

[24] P.-O. Chapuis, M. Laroche, S. Volz, and J.-J. Greffet, Appl. Phys. Lett. 92, 201906 (2008).

[25] A. Manjavacas and F. J. García de Abajo, Phys. Rev. B 86, 075466 (2012).

[26] P. Ben-Abdallah, S. A. Biehs, and K. Joulain, Phys. Rev. Lett. 107, 114301 (2011).

[27] P. Ben-Abdallah, R. Messina, S. A. Biehs, M. Tschikin, K. Joulain, and C. Henkel, Phys. Rev. Lett. 111, 174301 (2013).

[28] M. Nikbakht, Phys. Rev. B 96, 125436 (2017).

[29] J. Dong, J. M. Zhao, and L. H. Liu, J. Quant. Spectrosc. Radiat. Transfer 197, 114 (2017).
[30] M. Krüger, G. Bimonte, T. Emig, and M. Kardar, Phys. Rev. B 86, 115423 (2012).

[31] B. Müller, R. Incardone, M. Antezza, T. Emig, and M. Krüger, Phys. Rev. B 95, 085413 (2017).

[32] L. Zhu, Y. Guo, and S. Fan, Phys. Rev. B 97, 094302 (2018).

[33] B. Czapla and A. Narayanaswamy, J. Quant. Spectrosc. Radiat. Transfer 227, 4 (2019).

[34] S. Edalatpour and M. Francoeur, J. Quant. Spectrosc. Radiat. Transfer 133, 364 (2014).

[35] A. W. Rodriguez, M. T. H. Reid, and S. G. Johnson, Phys. Rev. B 86, 220302 (2012).

[36] D. Becerril and C. Noguez, Phys. Rev. B 99, 045418 (2019).

[37] J. Dong, J. Zhao, and L. Liu, Phys. Rev. B 95, 125411 (2017).

[38] J. Chen, C. Y. Zhao, and B. X. Wang, J. Quant. Spectrosc. Radiat. Transfer 219, 304 (2018).

[39] C. M. Sorensen, Aerosol Sci. Technol. 35, 648 (2001).

[40] K. Skorupski, J. Mroczka, T. Wriedt, and N. Riefler, Physica A 404, 106 (2014).

[41] C. F. Bohren and D. R. Huffman, Absorption and Scattering of Light by Small Particles (Wiley, New York, 1983).

[42] G. W. Mulholland, C. F. Bohren, and K. A. Fuller, Langmuir 10, 2533 (1994).

[43] M. A. Ordal, R. J. Bell, R. W. Alexander, L. L. Long, and M. R. Querry, Appl. Opt. 24, 4493 (1985).

[44] M. Antezza and Y. Castin, Phys. Rev. Lett. 103, 123903 (2009). 